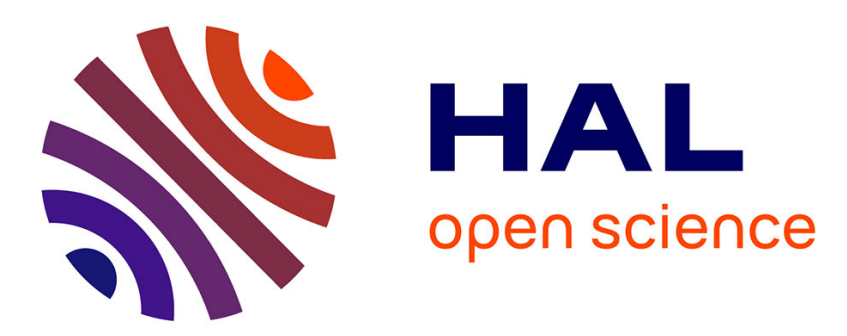

\title{
A decentralized Second Order Sliding-Mode control of multi-agent system with communication delay
}

Rubén Hernández, Vincent Léchappé, Jesus de Leon, Franck Plestan

\section{To cite this version:}

Rubén Hernández, Vincent Léchappé, Jesus de Leon, Franck Plestan. A decentralized Second Order Sliding-Mode control of multi-agent system with communication delay. Variable Structure System, Jun 2016, Nanjing, China. 10.1109/VSS.2016.7506883 • hal-01456471

\section{HAL Id: hal-01456471 \\ https://hal.science/hal-01456471}

Submitted on 5 Feb 2017

HAL is a multi-disciplinary open access archive for the deposit and dissemination of scientific research documents, whether they are published or not. The documents may come from teaching and research institutions in France or abroad, or from public or private research centers.
L'archive ouverte pluridisciplinaire HAL, est destinée au dépôt et à la diffusion de documents scientifiques de niveau recherche, publiés ou non, émanant des établissements d'enseignement et de recherche français ou étrangers, des laboratoires publics ou privés. 


\section{A Decentralized Second Order Sliding-Mode Control of Multi-Agent System with Communication Delay}

\author{
Rubén Hernández and Jesús De León \\ Facultad de Ingeniería \\ Mecánica y Eléctrica \\ Universidad Autónoma de Nuevo León \\ Nuevo León, México \\ Email: rhernandeza6@gmail.com \\ and drjleon@gmail.com
}

\author{
Vincent Léchappé \\ Laucala campus \\ University of the South Pacific \\ Suva, Fiji \\ Email: vincentlechappe@gmail.com
}

\author{
Franck Plestan \\ Institut de Recherche \\ en Communications \\ et Cybernétique de Nantes \\ Ecole Centrale de Nantes \\ Nantes, France \\ Telephone: (024) 037-6914 \\ Fax: (024) 074-7406
}

\begin{abstract}
This paper addresses the formation control problem of a multi-agent system (MAS) under the leader-follower scheme. Thus, the followers must to keep a desired relative distance with respect to the leader trajectory against known and constant communication delay. Thus, in order to ensured the coordinated behavior and formation control against parametric uncertainties and external disturbances, a decentralized control approach based on a state predictor and a Second Order Sliding Mode control is proposed. Sufficient conditions are provided to guarantee the stability of the closed-loop system. Finally, simulation results illustrate the effectiveness of the proposed method.
\end{abstract}

\section{INTRODUCTION}

Motion control in MAS has been trendy topic in the last years, due that there are tasks that can be performed more efficiently by a MAS instead of by a single agent. A MAS is a set of autonomous units that interact with each other in a common environment under cooperative or coordinated schemes. In coordinated schemes there are only interconnections from the leader or dominant system to rest, while in cooperative schemes there are interconnections among all the agents, such that they have influence on the combined dynamics [7]. Thus, an example of MAS is a group of mobile robots, that interact in order to perform tasks such as surveillance, exploration, or to move large objects, where it is required to keep a desired formation with respect to global ${ }^{1}$ reference given by the leader robot. A formation is a set of coordinates that defines a geometric structure in a coordinate space. Thus, leader-follower formation objective is such that followers form a desired formation given by its own desired relative distances with respect to the leader trajectory, where the leader is independent of their followers. This objective can be addressed by a decentralized approach where only local information from the neighbors is required [5]. Several approaches based on decentralized control have

\footnotetext{
1 "global" is used in this paper to refer to variables that involve to the MAS and are denoted by typeface in bold, whereas "local" is used to refer to variables related to each particular agent.
}

been proposed in order to tackle the formation control problem. For example in [1] a leader-followers based formation controller is presented for a group of non disturbed unicycle robots. In this control approach it is assumed that the linearization is possible, this being a disadvantage against parametric uncertainties. On the other hand, traditional sliding modes control is used in many applications including formation and consensus control, as it enables accuracy tracking and robustness to disturbances and plant parameter variations. In [3] an adaptive sliding mode formation controller based on leader-follower approach is considered for a six degree of freedom (DOF) nonlinear systems. Under this approach, the upper bounds of uncertainties are estimated via neural networks to compensate the effects of unknown disturbances and modeling errors. Nevertheless, the main disadvantage in these works is chattering phenomenon. Approaches based on high-order sliding modes technique represent an interesting alternative, because chattering phenomenon is attenuated. In [8], the finite time consensus problem of disturbed secondorder MAS is addressed through Super Twisting Algorithm (STA) for the disturbance-rejection. However, it should be pointed out that in practical multi-agent system network time delay is unavoidable and is a factor that causes instability in MAS, and should be taken into account in consensus controller design. The communication delay is related to the information flow between agents [9]. In [11] a consensus protocol based on Smith Predictor, finite spectrum assignment method and the Arstein reduction is proposed with the aim to avoid the effect of large time delay. It should be mentioned that this control approach is not robust to model uncertainties and external disturbances.

In this work, the leader-follower formation control problem for a group of unicycle mobile robots with communication delays is addressed. In order to solve this problem, a decentralized formation control based on recent predictive method [13] and STA is proposed. Thus, the delay is compensated and robustness against parametric uncertainties and external 
disturbances is ensured. This paper is organized as follows: in Section II, a system description and mathematical model of a mobile robot are provided. Thus, a MAS model based on leader-follower approach is introduced. The synthesis of control scheme is derived in Section III, where a state predictor is presented in order to compensate delay effects. Then, a Super-Twisting Control design for driving trajectories of the mobile robots is addressed. Simulation results are given in Section IV with the aim to illustrate the feasibility of the proposed scheme. Finally, conclusions of this work are drawn.

\section{SYSTEM DESCRIPTION}

In this section, a system description is presented. In the first paragraph of this section, the mathematical model for a single agent is described. Thus, a global representation of MAS under leader-follower scheme is derived in the second paragraph.

\section{A. Unicycle mobile robot model}

The unicycle mobile robot represent an attractive combination of a simple wheel configuration with a high traction through pneumatic tires (see Fig. 1). The mathematical model without external disturbances [12] of the $i$-th robot for $i=$ $1,2, \ldots, N$; is defined as

$$
\dot{X}_{i}=f_{i}\left(X_{i}\right)+g\left(X_{i}\right) u_{i}, \quad Y_{i}=h_{i}\left(X_{i}\right),
$$

where $X_{i}=\left[x_{i}, y_{i}, v_{i}, \omega_{i}, \theta_{i}\right]^{T} \in \mathbb{R}^{5}$ denotes the state vector. The input vector is denoted by $u_{i}=\left[\begin{array}{ll}F_{i} & \tau_{i}\end{array}\right]^{T} \in \mathbb{R}^{2}$ and the terms $F_{i}, \tau_{i}$ correspond to the translation force and to the torque applied to guide the robot in the cartesian space, respectively. The position of the $i$-th robot in the cartesian space is a measurable function denoted by the hand position $h_{i}\left(X_{i}\right)=\left[\begin{array}{ll}x_{i} & y_{i}\end{array}\right]^{T} \in \mathbb{R}^{2}$ [12] and corresponds to system output. The functions $f_{i}\left(X_{i}\right), g_{i}\left(X_{i}\right)$ are smooth vector fields defined as

$$
f_{i}\left(X_{i}\right)=\left[\begin{array}{c}
v_{i} \mathrm{C}_{\theta_{i}}-l_{i} \omega_{i} \mathrm{~S}_{\theta_{i}} \\
v_{i} \mathrm{~S}_{\theta_{i}}+l_{i} \omega_{i} \mathrm{C}_{\theta_{i}} \\
0 \\
0 \\
\omega_{i}
\end{array}\right], \quad g_{i}\left(X_{i}\right)=\left[\begin{array}{cc}
0 & 0 \\
0 & 0 \\
\frac{1}{m_{i}} & 0 \\
0 & \frac{1}{I_{i}} \\
0 & 0
\end{array}\right],
$$

where the terms $\theta_{i}$ denoted the orientation, $v_{i}$ the linear speed, and $\omega_{i}$ the angular speed; $\mathrm{s}_{(\cdot)}$ and $\mathrm{C}_{(\cdot)}$ correspond to sine and cosine functions. Moreover, $m_{i}$ correspond to the mass and $I_{i}$ to the moment of inertia. In addition, it is considered that the center of mass coincide with the geometric center. On the other hand, system (1) has relative degree vector $\rho=$ $(2,2)$. Then, directed relationship between the output $Y_{i}$ and input $u_{i}$ is generated after the $2^{\text {nd }}$ derivative of the output as $\ddot{Y}_{i}=L_{f_{i}}^{2} h_{i}+L_{g_{i}} L_{f_{i}} h_{i} u_{i}$, where $L_{f_{i}} h_{i}$ is the Lie derivative of $h_{i}$ with respect to $f_{i}$ for $i=1, \ldots, N$; Thus, there exists a diffeomorphism $\Phi: \mathbb{R}^{5} \rightarrow \mathbb{R}^{5}$ defined as

$$
\Phi\left(X_{i}\right)=\left[\begin{array}{c}
h_{1 i}\left(X_{i}\right) \\
h_{2 i}\left(X_{i}\right) \\
L_{f} h_{1 i}\left(X_{i}\right) \\
L_{f} h_{2 i}\left(X_{i}\right) \\
\eta_{i}(t)
\end{array}\right]=\left[\begin{array}{c}
x_{i} \\
y_{i} \\
v_{i} \mathrm{C}_{\theta_{i}}-l_{i} \omega_{i} \mathrm{~S}_{\theta_{i}} \\
v_{i} \mathrm{~S}_{\theta_{i}}+l_{i} \omega_{i} \mathrm{C}_{\theta_{i}} \\
\theta_{i}
\end{array}\right]
$$

so that $X_{i}=\Phi^{-1}\left(\left[\zeta_{i}(t), \eta_{i}(t)\right]\right)$, for $\zeta_{i}(t)=$ $\left[\zeta_{1 i}(t), \zeta_{2 i}(t), \zeta_{3 i}(t), \zeta_{4 i}(t)\right]^{T} \in \mathbb{R}^{4}$ and $\eta_{i}(t) \in \mathbb{R}$. Note that, from (3) the vector $\left[\zeta_{1 i}(t), \zeta_{2 i}(t)\right]^{T}=\left[x_{i}, y_{i}\right]^{T}$ is the hand position and its derivative as $\left[\zeta_{3 i}(t), \zeta_{4 i}(t)\right]^{T}=\left[\dot{x}_{i}, \dot{y}_{i}\right]^{T}$. Thus, if the trajectories of (1) are perturbed by a bounded external input disturbance $d_{i}(t)$, the system can be written in transformated coordinates as

$$
\begin{gathered}
\dot{\zeta}_{i}(t)=A \zeta_{i}(t)+B\left[\alpha_{i}\left(\zeta_{i}, \eta_{i}\right)+\beta_{i}\left(\zeta_{i}, \eta_{i}\right)\left(u_{i}(t)+d_{i}(t)\right)\right] \\
\dot{\eta}_{i}(t)=\varphi_{i}\left(\zeta_{i}, \eta_{i}\right) \\
Y_{i}(t)=C \zeta_{i}(t), \quad i=1,2, \ldots, N
\end{gathered}
$$

where, the matrix $A, B$, and $C$ are given by

$$
\begin{aligned}
& A=\left[\begin{array}{llll}
0 & 0 & 1 & 0 \\
0 & 0 & 0 & 1 \\
0 & 0 & 0 & 0 \\
0 & 0 & 0 & 0
\end{array}\right], B=\left[\begin{array}{ll}
0 & 0 \\
0 & 0 \\
1 & 0 \\
0 & 1
\end{array}\right], \\
& C=\left[\begin{array}{llll}
1 & 0 & 0 & 0 \\
0 & 1 & 0 & 0
\end{array}\right],
\end{aligned}
$$

from (3), the matrix $\alpha_{i}\left(\zeta_{i}, \eta_{i}\right) \in \mathbb{R}^{2}, \beta_{i}\left(\zeta_{i}, \eta_{i}\right) \in \mathbb{R}^{2 \times 2}$ and $\varphi_{i}\left(\zeta_{i}, \eta_{i}\right) \in \mathbb{R}$ for $i=1,2, \ldots, N$; are continuous functions where " $(t) "$ it is omitted of its argument, and are defined as

$$
\begin{aligned}
\alpha_{i}\left(\Phi\left(X_{i}\right)\right) & =\left[\begin{array}{c}
-v_{i} \omega_{i} \mathrm{~s}_{\theta_{i}}-l_{i} \omega_{i}^{2} \mathrm{C}_{\theta_{i}} \\
v_{i} \omega_{i} \mathrm{C}_{\theta_{i}}-l_{i} \omega_{i}^{2} \mathrm{~s}_{\theta_{i}}
\end{array}\right], \\
\beta_{i}\left(\Phi\left(X_{i}\right)\right) & =\left[\begin{array}{cc}
\frac{1}{m_{i}} \mathrm{C}_{\theta_{i}} & -\frac{l_{i}}{I_{i}} \mathrm{~s}_{\theta_{i}} \\
\frac{1}{m_{i}} \mathrm{~s}_{\theta_{i}} & \frac{l_{i}}{I_{i}} \mathrm{C}_{\theta_{i}}
\end{array}\right], \\
\varphi_{i}\left(\Phi\left(X_{i}\right)\right) & =-\frac{1}{L_{i}} \dot{x}_{i} \mathrm{~s}_{\theta_{i}}+\frac{1}{L_{i}} \dot{y}_{i} \mathrm{C}_{\theta_{i}} .
\end{aligned}
$$

The unknown bounded function $\beta_{i}\left(\zeta_{i}, \eta_{i}\right)=\hat{\beta}_{i}\left(\zeta_{i}, \eta_{i}\right)+$ $\Delta \beta_{i}\left(\zeta_{i}, \eta_{i}\right)$ is invertible and positive definite for all $\zeta_{i}(t)$ and $t \in[0, \infty)$. The term $\hat{\beta}_{i}\left(\zeta_{i}, \eta_{i}\right)=L_{\mathcal{G}_{i}} L_{\mathcal{F}_{i}} h_{i}$ is the nominal part known of $\beta_{i}\left(\zeta_{i}, \eta_{i}\right)$, where the functions $\mathcal{F}_{i} \in \mathbb{R}^{5}$, $\mathcal{G}_{i} \in \mathbb{R}^{5 \times 2}$ are smooth vector fields (similar to (2)) with the nominal value of mass $\hat{m}_{i}$ and moment of inertia $\hat{I}_{i}$. The function $\Delta \beta_{i}\left(\zeta, \eta_{i}\right) \in \mathbb{R}^{2 \times 2}$ is a bounded perturbation with the same structure of $\beta_{i}\left(\zeta_{i}, \eta_{i}\right)$ in (8), and has unknown variations on mass $\Delta m_{i}$ and moment of inertia $\Delta I_{i}$. The equation (9) represent the internal dynamics which are rendered unobservable and uncontrollable by the transformation (3) [12]. The zero dynamic is stable, due to $\dot{\eta}_{i}=0$ for $\zeta_{3 i}, \zeta_{4 i}=0$ [12]. On the other hand, in order to provide adequate structure for the design of the proposed scheme, the control input $u_{i}$ is implemented by auxiliary control $\Omega_{i}(t) \in \mathbb{R}^{2}$ as

$$
u_{i}(t)=\hat{\beta}_{i}^{-1}\left(\zeta_{i}, \eta_{i}\right) \Omega_{i}(t),
$$

Now, the system (4) with the feedback (10) can be expressed as perturbed linear system:

$$
\dot{\zeta}_{i}(t)=A \zeta_{i}(t)+B \Omega_{i}(t)+B p_{i}(t),
$$

where the perturbation term $p_{i}(t) \in \mathbb{R}^{2}$ is,

$$
\begin{aligned}
p_{i}(t)= & \alpha_{i}\left(\Phi\left(X_{i}\right)\right)+\beta_{i}\left(\zeta_{i}, \eta_{i}\right) d_{i}(t) \\
& +\left(\beta_{i}\left(\zeta_{i}, \eta_{i}\right) \hat{\beta}_{i}^{-1}\left(\zeta_{i}, \eta_{i}\right)-I_{2}\right) \Omega_{i}(t) .
\end{aligned}
$$




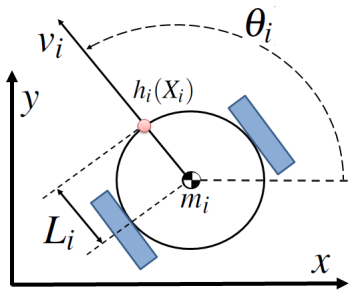

Figure 1. Differential-drive mobile robot.

In the next section, the MAS under leader-follower scheme will be formulated.

\section{B. MAS model based on Leader-Follower approach}

Let us define a set of $N$ linear systems described by (11) and (6), given by

$$
\begin{aligned}
\dot{\boldsymbol{\zeta}}(t) & =\left(I_{N} \otimes A\right) \boldsymbol{\zeta}(t)+\left(I_{N} \otimes B\right) \boldsymbol{\Omega}(t)+\left(I_{N} \otimes B\right) \boldsymbol{p}(t), \\
\boldsymbol{Y}(t) & =\left(I_{N} \otimes C\right) \boldsymbol{\zeta}(t),
\end{aligned}
$$

where operator $\otimes$ denotes the Kronecker product (or tensor product) [6]; $\boldsymbol{\zeta}(t)=\left[\zeta_{1}^{T}(t), \ldots, \zeta_{N}^{T}(t)\right]^{T}$ represents the global state vector, and its elements are the state vectors of the followers. The $\boldsymbol{p}(t)=\left[p_{1}(t), \ldots, p_{N}(t)\right]^{T}$ denotes the vector perturbation term and $\boldsymbol{\Omega}(t)=\left[\Omega_{1}^{T}(t), \ldots, \Omega_{N}^{T}(t)\right]^{T}$ is the formation control vector.

The multi-agent system follower (13) must track the leader trajectory, that is described in global form as

$$
\dot{\boldsymbol{\zeta}}_{o}(t)=\left(I_{N} \otimes A\right) \boldsymbol{\zeta}_{o}(t)+\left(I_{N} \otimes B\right) \boldsymbol{\Omega}_{o}(t)+B \boldsymbol{p}_{o}(t),
$$

where the perturbation term $\boldsymbol{p}_{o}(t)=\mathbf{1}_{N} \otimes p_{o}(t)$ has the same structure than (12), and $\mathbf{1}_{N}$ is a $\mathrm{N}$ dimensional vector of ones. The state vector $\zeta_{o}(t)=\mathbf{1}_{N} \otimes \zeta_{o}(t)$ and $\zeta_{o}(t)=\left[\zeta_{1 o}(t), \zeta_{2 o}(t), \zeta_{3 o}(t), \zeta_{4 o}(t)\right]^{T}$ respect to position and speed in coordinated space; finally, the control input $\Omega_{o}(t)=1_{N} \otimes \Omega_{o}(t)$, where the vector $\Omega_{0}(t)$ is the leader input.

Assumption 1. The control signal $\Omega_{o}(t) \in \mathbb{R}^{2}$ is a locally integrable function at unbounded interval $[-r,+\infty[$.

Assumption 2. The unknown disturbance signal $p_{o}(t) \in \mathbb{R}^{2}$ is a locally integrable function at unbounded interval $\mathbb{R} \geq 0$ for all $t \geq 0$, with a $p_{\max } \geq 0$ as upper bound, i.e., $\left\|p_{o}(t)\right\| \leq p_{\max }<+\infty$.

A desired formation is a set of references that defines a geometric structure in a coordinate space. Thus, in order to keep a desired formation, each subsystems in (13) cooperate to keep a desired relative distance with respect to the leader trajectory (14). Desired relative distance for $i$-th robot is denoted by $\mathcal{L}_{1 i}(t)$ and $\mathcal{L}_{2 i}(t)$ on $x$-axis and $y$-axis, respectively, and satisfies the follow equation:

$$
\dot{\mathcal{L}}_{i}(t)=A \mathcal{L}_{i}(t)+\hat{B} \dot{\mathcal{L}}_{i}(t),
$$

where $\mathcal{L}_{i}(t)=\left[\mathcal{L}_{1 i}(t), \mathcal{L}_{2 i}(t), \dot{\mathcal{L}}_{1 i}(t), \dot{\mathcal{L}_{2 i}}(t)\right]^{T}$; the expression $\hat{B} \dot{\mathcal{L}}_{i}(t)=B\left[\begin{array}{ll}\ddot{\mathcal{L}}_{1 i}(t) & \ddot{\mathcal{L}}_{2 i}(t)\end{array}\right]^{T}$, where $\hat{B}=I_{4}-C^{T} C$. Thus, the expression (15) can be written in global form as

$$
\dot{\mathcal{L}}(t)=\left(I_{N} \otimes A\right) \mathcal{L}(t)+\left(I_{N} \otimes \hat{B}\right) \dot{\mathcal{L}}(t),
$$

that will be used in the sliding surface design (presented in the next section), where $\mathcal{L}(t)=\left[\mathcal{L}_{1}^{T}(t), \ldots, \mathcal{L}_{N}^{T}(t)\right]^{T}$. Now, the interconnection topology is given by network star configuration, where the leader trajectory is the centroid of the desired formation. However, only delayed leader information $\zeta_{o}(t-r)$ is available and the consensus objective is not ensured. Therefore, the prediction $\bar{\zeta}_{o}(t) \simeq \zeta_{o}(t+r)$ will be transmitted to provide the approximation of actual value $\zeta_{o}(t)$ for each robot (see Figure 2). The prediction of leader state is estimated thank to a state predictor, that will be introduced in the next section.

\section{Control Scheme}

In this section, a control scheme based on STA with a Robust Predictor (RP) is presented. In order to compensate time delay, the prediction of leader state is transmitted through communication channel. Thus, the approximate actual value arrive to the followers (see Figure 2). Therefore, the RP will be introduced in the first part of this section, subsequently the sliding surface and the STA are presented at the end of this section.

\section{A. State Predictor [13]}

Since the leader trajectory is delayed in communication channel, a RP will be implemented to calculate an estimation of the prediction of leader state. The prediction state of (14) without disturbance is denoted by $\mathcal{X}_{o}(t)=\mathbf{1}_{N} \otimes \mathcal{X}_{o}(t)$ and reads as

$$
\mathcal{X}_{o}(t)=e^{A r} \zeta_{o}(t)+\int_{t-r}^{t} e^{A(t-\Theta)} B \Omega_{o}(\Theta) d \Theta,
$$

Now, with the aim to improve the approximated prediction $\mathcal{X}_{o}(t)$, disturbances effect can be estimated from available state in (14) and previous information from (17) as

$$
\zeta_{o}(t)-\mathcal{X}_{o}(t-r)=\int_{t-r}^{t} e^{A(t-\Theta)} B p_{o}(\Theta) d \Theta,
$$

that is well defined for all $t \geq 0$. Finally, the RP is defined as

$$
\overline{\boldsymbol{\zeta}}_{o}(t)=\boldsymbol{\mathcal { X }}_{o}(t)+\boldsymbol{\zeta}_{o}(t)-\mathcal{X}_{o}(t-r),
$$

where the term $\zeta_{o}(t)-\mathcal{X}_{o}(t-r)=\mathbf{1}_{N} \otimes\left[\zeta_{o}(t)-\mathcal{X}_{o}(t-r)\right]$, and $\bar{\zeta}_{o}(t) \simeq \zeta_{o}(t+r)$.

Remark 1. The disturbance compensation in (18) depends on time delay, because only previous leader information is available. Then, the compensation disturbance in the prediction is given at time $(t-r)$.

\section{B. Super-Twisting Algorithm}

In this paragraph, a controller described in [2] is extended to a formation control of MAS with communication delay. In order to ensure the formation control based on consensus, the formation error $\boldsymbol{\varepsilon}(t)$ is defined as

$$
\boldsymbol{\varepsilon}(t)=\boldsymbol{\zeta}(t)-\overline{\boldsymbol{\zeta}}_{o}(t-r)-\boldsymbol{\mathcal { L }}(t) .
$$

Note that term $\boldsymbol{\zeta}(t)-\overline{\boldsymbol{\zeta}}_{o}(t-r)$ of (20) corresponds to the relative position of follower with respect to the leader. Thus, 


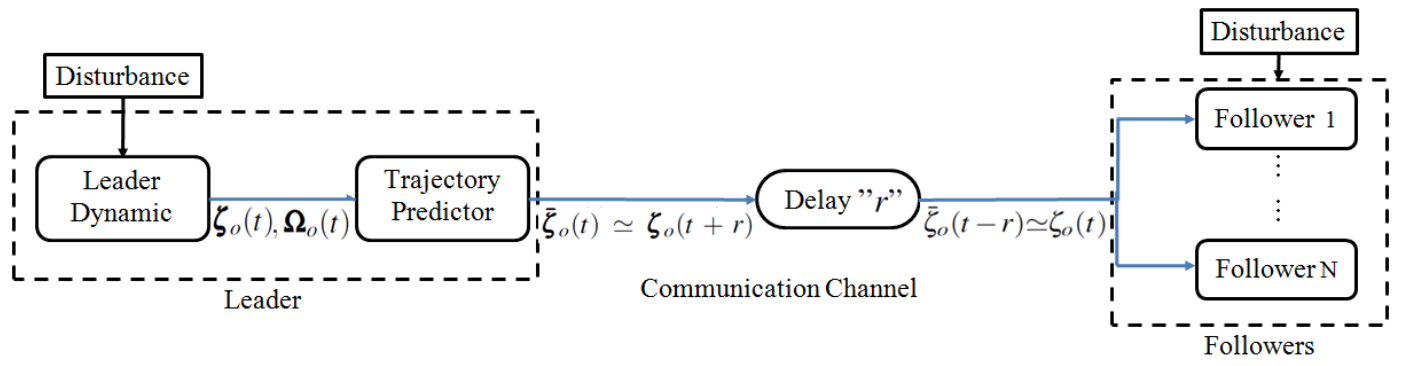

Figure 2. Transmission of state predicted trough channel communication.

if formation error $\varepsilon(t) \rightarrow \mathbf{0}_{2 N}$, the relative distances between leader and followers converge to desired relative distances, i.e., $\boldsymbol{\zeta}(t)-\overline{\boldsymbol{\zeta}}_{o}(t-r) \rightarrow \mathcal{L}(t)$. The vector $\overline{\boldsymbol{\zeta}}_{o}(t-r)=\mathbf{1}_{N} \otimes \bar{\zeta}_{o}(t-r) \simeq$ $\mathbf{1}_{N} \otimes \zeta_{o}(t)$. Now, considering that the speed is available, the chosen sliding variable is written as a function of the states predicted as

$$
\boldsymbol{S}(t)=\boldsymbol{\lambda}\left(I_{N} \otimes C\right) \boldsymbol{\varepsilon}(t)+\left(I_{N} \otimes C A\right) \boldsymbol{\varepsilon}(t),
$$

where $\boldsymbol{S}(t)=\left[s_{1}^{T}(t), \ldots, s_{N}^{T}(t)\right]^{T} \in \mathbb{R}^{2 N}$ is a global vector composed of local sliding variables of each agent $s_{i}(t)=$ $\left[s_{i 1}(t), s_{i 2}(t)\right]^{T} \in \mathbb{R}^{2}$ for $i=1, \ldots, N$; the first term in (21) is linked to the relative position error and the second term to its derivative. $\boldsymbol{\lambda}=\operatorname{diag}\left\{\lambda_{i}\right\} \in \mathbb{R}^{2 N \times 2 N}$ is a diagonal matrix gain, and $\lambda_{i} \in \mathbb{R}^{2 \times 2}$ as local diagonal matrix gain definite positive for $i=1, \ldots, N$; and is chosen such that all elements of $\boldsymbol{S}(t)$, $\dot{\boldsymbol{S}}(t)$ converges to 0 in finite time.

Remark 2. Taken $\mathbf{0}_{2 \mathrm{~N}} \in \mathbb{R}^{2 N}$ as a null vector of dimension $2 N$. The sliding variable (21) is designed taking into account that the desired compensated dynamics of the system (13) are achieved in the sliding mode $\boldsymbol{S}(t) \rightarrow \mathbf{0}_{2 N}$.

From (21), (13) and (19), the input-output dynamics of (21) result as

$$
\dot{\boldsymbol{S}}(t)=\boldsymbol{a}\left(\boldsymbol{\zeta}(t), \boldsymbol{\zeta}_{o}(t-r), t\right)+\boldsymbol{\Omega}(t),
$$

where, for simplicity, $\boldsymbol{a}\left(\boldsymbol{\zeta}(t), \boldsymbol{\zeta}_{o}(t-r), t\right)=\boldsymbol{a}$ and,

$$
\begin{aligned}
\boldsymbol{a}= & \boldsymbol{\lambda}\left(I_{N} \otimes C A\right)\left(\boldsymbol{\zeta}(t)-\boldsymbol{\zeta}_{o}(t-r)-\boldsymbol{\mathcal { L }}(t)\right)-\boldsymbol{\Omega}_{o}(t) \\
& -(1+r) \boldsymbol{\lambda} \boldsymbol{p}_{o}(t-r)+\left(I_{N} \otimes B^{T}\right) \dot{\mathcal{L}}(t)+\boldsymbol{p}(t) .
\end{aligned}
$$

Assumption 3. There exists unknown positive constant $\delta_{1}$ such that the derivative of the function $\boldsymbol{a}\left(\boldsymbol{\zeta}(t), \boldsymbol{\zeta}_{o}(t-r), t\right)$ is bounded as $\left\|\dot{\boldsymbol{a}}\left(\boldsymbol{\zeta}(t), \boldsymbol{\zeta}_{o}(t-r), t\right)\right\| \leq \delta_{1}$.

The problem is to drive the global sliding variable $\boldsymbol{S}(t)$ and $\dot{\boldsymbol{S}}(t)$ to $\mathbf{0}_{2 N}$ in finite time in presence of the bounded perturbation with the boundary $\delta_{1}>0$, where the argument "(t)" from sliding variable (21) and (22) will be omit. The robust control $\boldsymbol{\Omega}(t)=\boldsymbol{\Omega}$ is obtained using the following STA algorithm given by

$$
\boldsymbol{\Omega}=-\boldsymbol{K}_{1} \boldsymbol{D}_{s}^{1 / 2} \operatorname{sign}(\boldsymbol{S})+\nu, \quad \dot{\nu}=-\frac{1}{2} \boldsymbol{K}_{\mathbf{2}} \operatorname{sign}(\boldsymbol{S})
$$

where the function $\operatorname{sign}(S)$ returns a vector with the signs of the corresponding elements of $S \in \mathbb{R}^{2 N}$; the matrix $D_{s}=$ $\operatorname{diag}\left\{D_{s i}\right\}$. The submatrices $D_{s i}=\operatorname{diag}\left\{\left|s_{i} \iota\right|\right\}$ for $\iota=1,2$, where $s_{i 1}, s_{i 2}$ are elements of the local sliding surface of each agent $s_{i}=\left[s_{i 1}, s_{i 2}\right]^{T}$ for $i=1, \ldots, N$, i.e., $s_{i \iota} \in s_{i}$ for $\iota=1,2$; so that $s_{i} \in S$. The control gains are given by $\boldsymbol{K}_{1}=\operatorname{diag}\left\{k_{1 i}\right\}$ and $\boldsymbol{K}_{2}=\operatorname{diag}\left\{k_{2 i}\right\}$, where all submatrices $k_{1 i}, k_{2 i} \in \mathbb{R}^{2 \times 2}$ are diagonal matrices, for $i=1, \ldots, N$. From the system (22) and (23), the closed loop system is given by

$$
\dot{\boldsymbol{S}}=\boldsymbol{a}-\boldsymbol{K}_{1} \varsigma_{1}+\nu, \quad \dot{\nu}=-\frac{1}{2} \boldsymbol{K}_{2} \operatorname{sign}(\boldsymbol{S}) .
$$

In that case, stability criteria are given in the following theorem.

Theorem 1: Consider the system (13) and (21) in closedloop with control (23) and references given by state predictor (19). Then, from the assumptions 1,2 and 3, finite time convergence of $S$ and $\dot{S}$ is provided under STA to 2sliding mode for a unknown bound $\delta_{1}>0$ and given initial conditions $\varsigma(0)$ and $\boldsymbol{S}(0)$, if the control gains satisfies the matrix inequalities $\left\|\boldsymbol{K}_{1}\right\|>0$ and $\lambda_{j}\left(\boldsymbol{K}_{2}\right)>2 \delta_{1}$, where the unknown bounded function $0<\left\|\boldsymbol{\varrho}\left(\boldsymbol{\zeta}(t), \boldsymbol{\zeta}_{o}(t-r), t\right)\right\| \leq \delta_{1}$, and $\lambda_{j}\left(\boldsymbol{K}_{2}\right)$ denotes the real part of the eigenvalues of the matrix $\boldsymbol{K}_{2}$ for $j=1, \ldots, 2 N$.

Proof: Consider the following vector [10]

$$
\varsigma=\left[\varsigma_{1}^{T}, \varsigma_{2}^{T}\right]^{T}=\left[\operatorname{sign}(\boldsymbol{S})^{T} \boldsymbol{D}_{s}^{1 / 2}, \boldsymbol{a}^{T}+\nu^{T}\right]^{T} .
$$

Then, system (24) can be written as

$$
\dot{\varsigma}=\frac{1}{2}\left[\begin{array}{ll}
-\boldsymbol{D}_{s}^{-1 / 2} \boldsymbol{K}_{1} & \boldsymbol{D}_{s}^{-1 / 2} \\
-\boldsymbol{D}_{s}^{-1 / 2} \boldsymbol{K}_{2} & \mathbf{0}_{2 \mathrm{~N} \times 2 \mathrm{~N}}
\end{array}\right] \varsigma+\left[\begin{array}{l}
0 \\
1
\end{array}\right] \otimes \dot{\boldsymbol{a}},
$$

Furthermore, in similar way than [10] and due to the Assumption 5 , the boundary of the uncertain function $\dot{\boldsymbol{a}}=$ $\frac{1}{2} \boldsymbol{D}_{s}^{-1 / 2} \varrho\left(\zeta(t), \boldsymbol{\zeta}_{o}(t-r), t\right) \varsigma_{1}$ exists. In that case, the system (26) can be rewritten as follows

$$
\dot{\varsigma}=\frac{1}{2}\left(I_{2} \otimes D_{s}\right)^{-1 / 2} \boldsymbol{A} \varsigma,
$$

taken $\boldsymbol{A}=\left[\begin{array}{cc}-\boldsymbol{K}_{1} & I_{2 N} \\ -\left(\boldsymbol{K}_{2}-2 \varrho\left(\boldsymbol{\zeta}(t), \boldsymbol{\zeta}_{o}(t-r), t\right)\right) & \mathbf{0}_{2 \mathrm{~N} \times 2 \mathrm{~N}}\end{array}\right]$, where $\left\|s_{1}\right\| \geq\left\|\left(I_{2} \otimes \boldsymbol{D}_{s}\right)\right\|^{1 / 2}=\left\|\boldsymbol{D}_{s}\right\|^{1 / 2}$ and the matrix $\boldsymbol{K}_{2}-2 \boldsymbol{\varrho}\left(\boldsymbol{\zeta}(t), \boldsymbol{\zeta}_{o}(t-r), t\right)>0$ if all submatrix are definite positive too. Now, consider the quadratic function as a strict Lyapunov candidate function for (23), such that

$$
V=\varsigma^{T} \boldsymbol{P} \varsigma,
$$


where $\boldsymbol{P}=\boldsymbol{P}^{T}>0$ is a constant, symmetric and positive matrix. Now, the derivative of strict Lyapunov function result

$$
\dot{V} \leq\left\|\boldsymbol{D}_{s}\right\|^{-1 / 2} \varsigma^{T}\left[\boldsymbol{A}^{T} \boldsymbol{P}+\boldsymbol{P} \boldsymbol{A}\right] \varsigma .
$$

Thus, since the matrix $A$ is Hurwitz, the matrix $\boldsymbol{P}=\boldsymbol{P}^{T}>0$ is an unique solution that satisfies the Algebraic Lyapunov Equation $\left[\boldsymbol{A}^{T} \boldsymbol{P}+\boldsymbol{P} \boldsymbol{A}\right]=-\tilde{Q}$ and $\dot{V}$ can be written as

$$
\dot{V} \leq-\left\|\boldsymbol{D}_{s}\right\|^{-1 / 2} \lambda_{\operatorname{Min}}(\tilde{Q})\|\varsigma\|^{2},
$$

taken $\lambda_{\operatorname{Min}}(\tilde{Q})$ as the minimum eigenvalue of the matrix $\tilde{Q}$. On the other hand, since $\|\varsigma\| \geq\left\|D_{s}\right\|^{1 / 2}$ it is true that $-\|\varsigma\| \geq$ $-\left\|\boldsymbol{D}_{s}\right\|^{-1 / 2}\|\varsigma\|^{2}$, then one gets that $\dot{V} \leq-\lambda_{\text {Min }}(\tilde{Q})\|\varsigma\|$. Now, since that $V \leq \lambda_{\text {Max }}(\boldsymbol{P})\|\varsigma\|^{2}$, taken $\lambda_{\text {Max }}(\boldsymbol{P})$ as the minimum eigenvalue of the matrix $\boldsymbol{P}$, this leads to show that

$$
\dot{V} \leq-\frac{\lambda_{\operatorname{Min}}(\tilde{Q})}{\lambda_{\text {Max }}^{1 / 2}(\boldsymbol{P})} V^{1 / 2} .
$$

Therefore, since $\varsigma_{1}$ and $\varsigma_{2}$ convergence in finite time, convergence of $\boldsymbol{S}$ and $\dot{S}$ in finite time is ensured.

\section{Simulations}

In this section, simulation results are provided to illustrate the feasibility and the performance of the proposed methodology. In order to compare the performance of the proposed scheme, standard predictor [14] with STA has been also considered. System and control scheme have been developed with MATLAB/Simulink environment, using Runge-Kutta solver with an integration step of $0.001 \mathrm{~s}$. The MAS is composed by three unicycle robots (one leader and two followers), where the system parameters are given in the Table I. Note that simulation are made with parametric uncertainties in order to evaluated the robustness of proposed approach (for example, mass of the system $m_{i}=0.2 \mathrm{~kg}$ [see Table I], whereas nominal mass $\hat{m}_{i}=0.16 \mathrm{~kg}$ in the controller [see Table II]). The simultaneous time-varying external disturbances applied to each robot are displayed in Figure 3. On the other hand, According to leader-follower formation control approach, the follower robots must keep a desired relative distance with respect to the leader (see Figure 4), where the control of the leader and followers $\Omega_{i}(t)$ are based on (23) for $i=o, 1,2$. In order to evaluate the performance of the STA with RP, STA using standard predictor (SP) is tested. A time delay $r=0.4$ seconds in flow leader information is considered. The norm of prediction error for SP and RP are displayed on Figure 5Top; it is clear to see that RP has a higher accuracy than SP. The simulation results are shown in Figure 5-Bottom, where the norms of formation error for each approach $(\mathrm{STA}+\mathrm{SP}$ and STA+RP) are displayed. The trajectories of robots are shown on Figures 7-6. From the graphs, it appears that formation control is ensured with high accuracy with the STA+RP scheme versus external disturbances and parametric uncertainties. Thus, the tracking error is smaller with for $\mathrm{STA}+\mathrm{RP}$ than with STA+SP.
Table I

MODEL PARAMETERS FOR $i=o, 1,2$

\begin{tabular}{|c||c||c|}
\hline Parameter & value & Description \\
\hline$m_{i}$ & $0.2 \mathrm{~kg}$ & Mass of the system \\
\hline$I_{i}$ & $6.89 \times 10^{-5} \mathrm{kgm}{ }^{2}$ & Moment of Inertia \\
\hline$l_{i}$ & $0.045 \mathrm{~m}$ & Distance from center of mass \\
\hline
\end{tabular}

Table II

PARAMETERS OF CONTROL SCHEME FOR $i=o, 1,2$.

\begin{tabular}{|c||c||c|}
\hline Parameter & value & Description \\
\hline$r$ & $0.4 \mathrm{sec}$ & Communication time delay \\
\hline$\hat{m}_{i}$ & $0.16 \mathrm{~kg}$ & Nominal Mass of the system \\
\hline$\hat{I}_{i}$ & $1.2 \times 10^{-4} \mathrm{kgm}{ }^{2}$ & Nominal Moment of Inertia \\
\hline $\boldsymbol{K}_{\iota}$ & $100 I_{4}$ & Control gain for $\iota=1,2$ \\
\hline $\boldsymbol{\lambda}$ & $I_{4}$ & Sliding gain \\
\hline
\end{tabular}
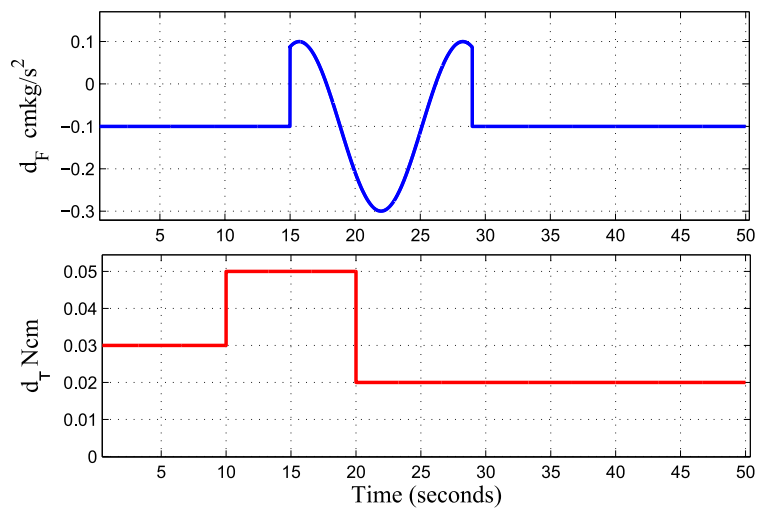

Figure 3. Input disturbances for all agents, where force $d_{F_{i}} \in d_{i}(t)$ and torque $d_{\tau_{i}} \in d_{i}(t)$ concerns to $41.5 \%$ of the input $F_{i}$ and $92.91 \%$ of the input $\tau_{i}$, respectively, for $i \in\{o, 1,2\}$.

\section{Time (seconds)}
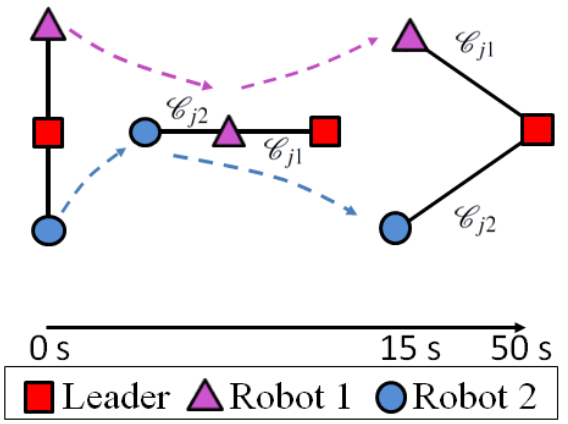

Figure 4. General scheme of desired formations with respect leader trajectory at different interval times, where $j=1,2$; denote $x$ and $y$ axis, respectively.

\section{CONCLusion}

In this work, the leader follower formation control problem with time delay communication has been considered. Thus, a control strategy combining a robust state predictor and super 

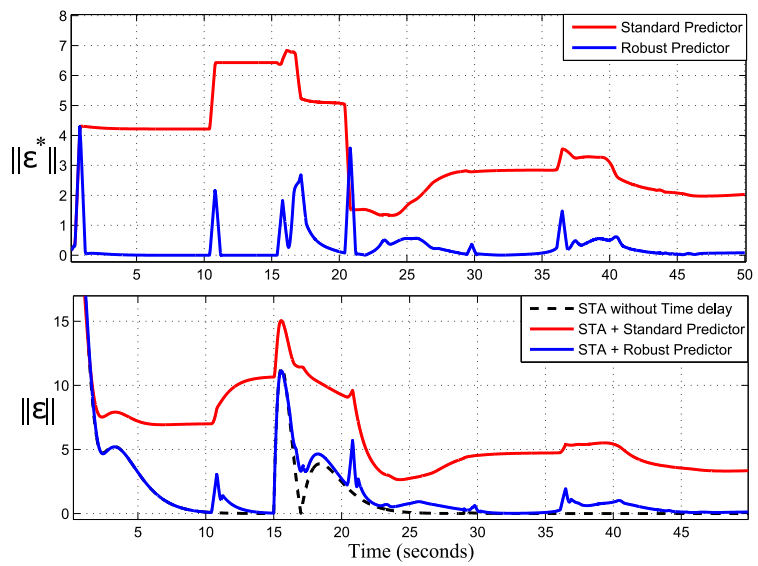

Figure 5. Comparison of error norm of the leader state prediction (Top) for $\varepsilon^{*}(t)=\boldsymbol{\zeta}_{o}(t)-\overline{\boldsymbol{\zeta}}_{o}(t-r)$ and $r=0.4$ seconds. Comparison of the norm of formation error (Bottom) for delay r $=0.4$ seconds, with $\boldsymbol{\varepsilon}(t)=\boldsymbol{\zeta}(t)-\boldsymbol{\zeta}_{o}(t)-$ $\mathcal{L}(t)$.
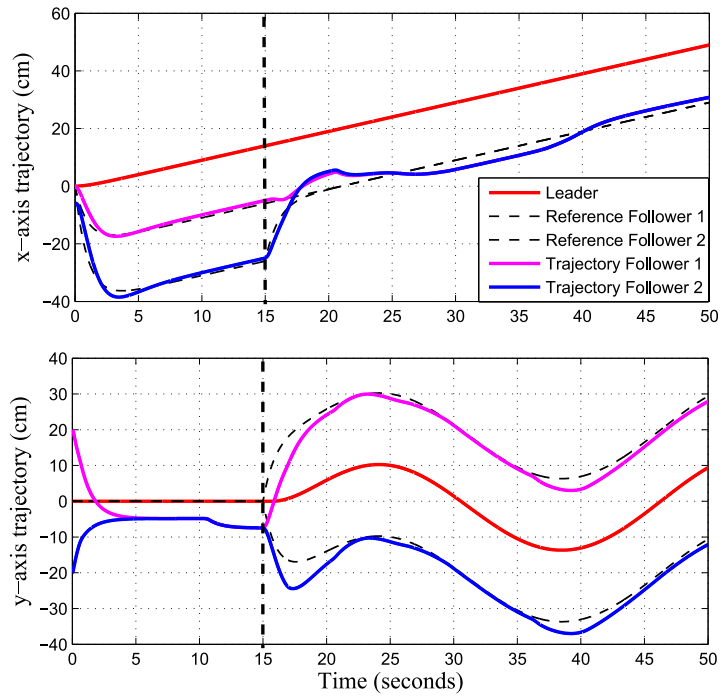

Figure 6. MAS trajectories with STA + SP, where switching of formation is denoted by vertical dashed line at 15 seconds

twisting algorithm has been proposed. On the other hand, since a good prediction plays an important role in consensus, the predicted leader trajectory is estimated by a robust state predictor. Therefore, dynamics of state predictor is treated as disturbance term in the input-output dynamics of sliding surface. Thus, in order to ensure finite time convergence, sufficient conditions of stability have been presented. Finally, simulation results demonstrated the feasibility and robustness of the proposed scheme.

\section{FUTURE WORKS}

This work present some opportunities for extending, for example: evaluate the proposed scheme in experimental way; extend the result to adaptive sliding mode control; include the
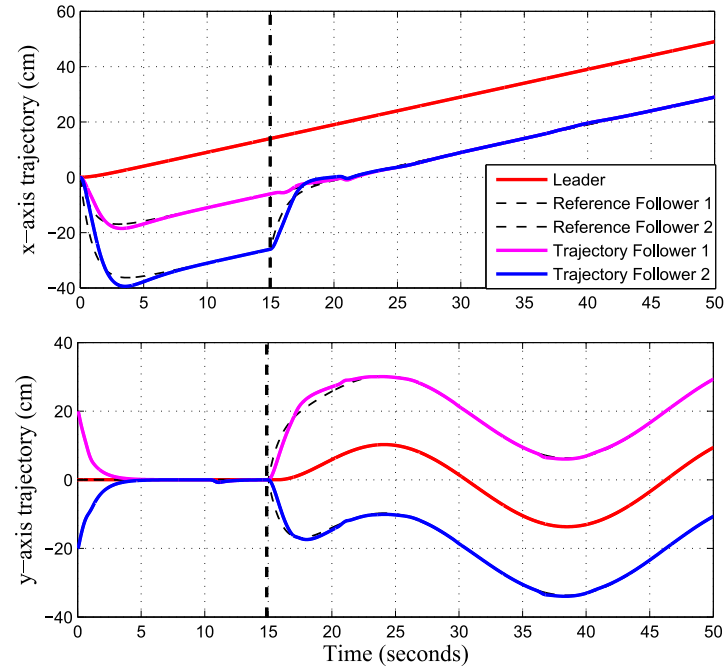

Figure 7. MAS trajectories with STA + RP, where switching of formation is denoted by vertical dashed line at 15 seconds

interactions among followers; and addressing the consensus problem for time-varying delay.

\section{REFERENCES}

[1] A. S. Brandao and M. Sarcinelli and R. Carelli and T. Freire, "Decentralized control of Leader-Follower formations mobile robots with obstacle avoidance", in Proc. of IEEE International conference on Mechatronics. Málaga, Spain, April 2009, pp. 4244-4195.

[2] A. Levant, "Sliding order and sliding accuracy in sliding mode control", International Journal of Control. vol 58, pp. 1247-1263, 1993.

[3] J. Bae, Y. Kim, "Adaptive controller design for spacecraft formation flying using sliding mode controller and neural networks", Journal of the Franklin Institute. vol 349, no 4, pp. 578 - 603, 2012.

[4] S. Boyd and L. El Ghaou and E. Feron and V. Balakrishnan "Linear Matrix Inequalities in System and Control Theory", SIAM Studies in Applied Mathematics. USA, 1994.

[5] C. De La Cruz and R. Carelli, "Dynamic model based formation control and obstacle avoidance of multi-robot systems", Robotica. vol 26, no 4, pp. 345-356, 2008.

[6] R. A. Horn and C.R. Jhonson, Matrix analysis, Cambridge University Press, Cambridge. 1985.

[7] H. Nijmeijer, A. Rodriguez, Synchronization of Mechanical Systems, World Scientific series on NonLinear Science. 2003.

[8] S. Yu and X. Long, "Finite-time consensus for second-order multi-agent systems with disturbances by integral sliding mode", Automatica. vol 54, no 1, pp. 158 - 165, 2015.

[9] C. Liu and F. Liu, "Consensus Problem of second-Order Multi-Agent Systems with Input Delay", International Journal of Information and systems Sciences. vol 7, no 3, pp. 175-191, 2011.

[10] Y. Shtessel and M. Taleb and F. Plestan, "A novel adaptive-gain supertwisting sliding mode controller: methodology and application", Automatica. vol 48, no 5, pp. 759-769, 2012.

[11] Z. Wang, J. Xu and H. Zhang, ”Consensus Seeking for Discrete-time Multi-agent Systems with Communication Delay", IEEE/CAA JOURNAL OF AUTOMATICA. vol 2, no 2, pp. $151-157,2015$.

[12] J.R.T. Lawton and R.W. Beard and B.J. Young "A decentralized approach to formation maneuvers", IEEE Transactions on Robotics and Automation. vol 19, no 6, pp. 933-941, 2003.

[13] V. Léchappé, E. Moulay, F. Plestan, A. Glumineau, A. Chriette "New Predictive Scheme for the control of LTI Systems With Input Delay and Unknown Disturbances", Automatica. vol 52, pp. 179-184, 2015.

[14] Z. Andrzej and W. Andrzej "Finite Spectrum Assignment Problem for Systems with Delays", IEEE Transactions On Automatic Control. vol 24, pp. 541-553, 1979. 